MATHEMATICS OF COMPUTATION

Volume 66, Number 217, January 1997, Pages 411-415

S 0025-5718(97)00779-5

\title{
CONSTRUCTION OF HIGH-RANK ELLIPTIC CURVES WITH A NONTRIVIAL TORSION POINT
}

\author{
KOH-ICHI NAGAO
}

\begin{abstract}
We construct a family of infinitely many elliptic curves over $\mathbb{Q}$ with a nontrivial rational 2-torsion point and with rank $\geq 6$, which is parametrized by the rational points of an elliptic curve of rank $\geq 1$.
\end{abstract}

\section{INTRODUCTION}

The problem of constructing high-rank elliptic curves over $\mathbb{Q}$ with a nontrivial torsion point has been studied by several people. Among them, Kretschmer [1] found an example of rank $\geq 10$ and Zimmer and Schneiders [6] found two examples of rank $\geq 11$. Regarding the problem of constructing infinitely many such curves, Mestre [3] found elliptic curves of the form $y^{2}=x^{3}+k x$ (where $(0,0)$ is a 2-torsion point) with rank $\geq 4$. In this note, we show the following.

Theorem 1. There are infinitely many elliptic curves over $\mathbb{Q}$ with a nontrivial 2 -torsion point and with rank $\geq 6$.

\section{The Curve $Y^{2}=a X^{4}+b X^{2}+c$}

In this note, high-rank elliptic curves of the form $Y^{2}=a X^{4}+b X+c$ are treated. First, we show that curves of this form have nontrivial 2-torsion points.

Lemma 2.1. Let $E: Y^{2}=a X^{4}+b X^{2}+c$ be a curve of genus one over a field $K$. Assume that $E$ has a $K$-rational point $(x, y)$ and regard $E$ as an elliptic curve whose group structure is given by $(x, y)$ as origin. Then one has $2(-x,-y)=0$.

Sketch of the proof. We denote the two points at infinity on $E$ by $\infty$ and $\infty^{\prime}$. More precisely, $\infty$ and $\infty^{\prime}$ are written as $(0, \sqrt{a}),(0,-\sqrt{a})$, respectively, on the dual model of $E$ given by the equation $Y^{2}=c X^{4}+b X^{2}+a$. Then we have

(1) $2 \infty-2 \infty^{\prime}=\operatorname{div}\left(-Y+\sqrt{a} X^{2}+\frac{b}{2 \sqrt{a}}\right) \sim 0$,

(2) $(x, y)+(x,-y)-\infty-\infty^{\prime}=\operatorname{div}(X-x) \sim 0$,

(3) $(x,-y)+(-x,-y)-2 \infty=\operatorname{div}\left(Y+\sqrt{a} X^{2}-y+\sqrt{a} x^{2}\right) \sim 0$,

where the symbol $\sim$ means the relation of rational equivalence class of divisors. By eliminating $(x,-y)$ from (2) and (3), we have

$$
(-x,-y)-(x, y) \sim \infty-\infty^{\prime},
$$

Received by the editor June 16, 1994 and, in revised form, November 1, 1994 and November 13, 1995.

1991 Mathematics Subject Classification. Primary 11G05, 11D25; Secondary 11Y50.

Key words and phrases. Elliptic curve. 
and hence we obtain

$$
2(-x,-y)-2(x, y) \sim 2 \infty-2 \infty^{\prime} \sim 0,
$$

which completes the proof.

In $\S 3$, we will construct an elliptic curve over $\mathbb{Q}(T)$ of the form $\mathcal{E}: Y^{2}=a(T) X^{4}+$ $b(T) X^{2}+c(T)$, which contains at least six $\mathbb{Q}(T)$-rational points $P_{1}, \ldots, P_{6}$. Further, we consider $\mathcal{E}$ as a curve defined over the function field $\mathbb{Q}(C)$, where $C$ is the curve defined by the equation $S^{2}=a(T)$. So the two points $\infty$ and $\infty^{\prime}$ at infinity of $\mathcal{E}$ become $\mathbb{Q}(C)$-rational points and we can choose the point $\infty$ as the origin. We know the point $\infty^{\prime}$ is a nontrivial 2-division point, and we can use all six points $P_{1}, \ldots, P_{6}$ to obtain independent points. It is remarked that a rational point $p=(t, s)$ on the curve $C$ gives rise to an elliptic curve over $\mathbb{Q}$, which is obtained from $\mathcal{E}$ by the specialization $(T, S) \rightarrow(t, s)$. Thus, if $C$ has infinitely many rational points, we can obtain infinitely many elliptic curves over $\mathbb{Q}$ with a nontrivial 2 -torsion point and rank $\geq 6$.

\section{Construction}

For any 6-tuple $A=\left(a_{1}, a_{2}, a_{3}, a_{4}, a_{5}, a_{6}\right) \in \mathbb{A}^{6}(\mathbb{Q}(T))$, let

$$
p_{A}(X)=\left(X^{2}-a_{1}^{2}\right)\left(X^{2}-a_{2}^{2}\right)\left(X^{2}-a_{3}^{2}\right)\left(X^{2}-a_{4}^{2}\right)\left(X^{2}-a_{5}^{2}\right)\left(X^{2}-a_{6}^{2}\right) \in \mathbb{Q}(T)[X] .
$$

Then we see easily that there are uniquely determined (up to the signature of $r_{A}$ ) polynomials $g_{A}(X), r_{A}(X) \in \mathbb{Q}(T)[X]$ satisfying $\operatorname{deg} g_{A}(X)=6, \operatorname{deg} r_{A}(X) \leq 4$ and $p_{A}(X)=g_{A}(X)^{2}-r_{A}(X)$. (We note that $g_{A}(X)$ and $r_{A}(X)$ are contained in $\mathbb{Q}(T)\left[X^{2}\right]$.) In this note, we only treat the case when $\operatorname{deg} r_{A}(X)$ is 4 and the equation $r_{A}(X)=0$ has no double root. Then the curve $Y^{2}=r_{A}(X)$ is an elliptic curve over $\mathbb{Q}(T)$, which is denoted by $\mathcal{E}_{A}$, and contains the six $\mathbb{Q}(T)$-rational points $P_{i}=\left(a_{i}, g_{A}\left(a_{i}\right)\right)(i=1, \ldots, 6)$.

By Lemma 2.1, we see that $\mathcal{E}_{A}$ is an elliptic curve over $\mathbb{Q}(T)$ with nontrivial 2 -torsion points since $r_{A}(X)$ is an element of $\mathbb{Q}(T)\left[X^{2}\right]$. When $A$ is of the form $\left( \pm T+\alpha_{1}, \ldots, \pm T+\alpha_{6}\right) \quad\left(\alpha_{i} \in \mathbb{Q}\right)$, the coefficient of $X^{4}$ in $r_{A}(X)$ seems to be (however we cannot prove it) a quartic polynomial of $T$, which will be important for our purpose.

Thus we consider the case $A=(T+1, T+2, T+3,-T+5,-T+6,-T+9)$. Then the equation of $\mathcal{E}=\mathcal{E}_{A}$ is written as

$$
\begin{aligned}
& Y^{2}=4\left(\left(-311 T^{4}-2814 T^{3}+58104 T^{2}-239744 T+297024\right) X^{4}\right. \\
& +\left(622 T^{6}-1848 T^{5}+2380 T^{4}-90410 T^{3}-6696 T^{2}+2080960 T-3928704\right) X^{2} \\
& -311 T^{8}+4662 T^{7}-4288 T^{6}-171446 T^{5}+410752 T^{4} \\
& \left.+2203272 T^{3}-5965776 T^{2}-10364480 T+28872256\right)
\end{aligned}
$$

and $P_{i}$ are as follows:

$$
\begin{aligned}
& P_{1}=\left(T+1,2\left(-200 T^{3}+711 T^{2}+1512 T-5024\right)\right), \\
& P_{2}=\left(T+2,4\left(-73 T^{3}+192 T^{2}+714 T-2116\right)\right), \\
& P_{3}=\left(T+3,2\left(12 T^{3}+323 T^{2}+304 T-4192\right)\right), \\
& P_{4}=\left(-T+5,2\left(316 T^{3}-3165 T^{2}+10080 T-10784\right)\right), \\
& P_{5}=\left(-T+6,4\left(159 T^{3}-1832 T^{2}+6902 T-8252\right)\right), \\
& P_{6}=\left(-T+9,2\left(-300 T^{3}+5411 T^{2}-27128 T+40736\right)\right) .
\end{aligned}
$$


Let us consider the elliptic curve

$$
C: S^{2}=-311 T^{4}-2814 T^{3}+58104 T^{2}-239744 T+297024
$$

in the $(T, S)$-plane.

Lemma 3.1. The curve $C$ contains infinitely many rational points.

Proof. By a direct calculation, we see that $C$ has rational points whose $T$ coordinates are $-4,-8 / 3,-13 / 4,16 / 5,24 / 5,20 / 7,37 / 8,29 / 12,43 / 12,32 / 13,232 / 47$, $272 / 79,-230 / 113$. By the theorem of Mazur [2], stating that the number of torsion points of an elliptic curve over $\mathbb{Q}$ is $\leq 16$, we see that $C$ has infinitely many rational points since $C$ has more than 26 rational points.

Proposition 3.1. The points $P_{1}, P_{2}, \ldots, P_{6}$ are independent $\mathbb{Q}(C)$-rational points when the group structure is given by $\infty$ as origin.

We give the proof of Proposition 3.1 in the next section. Now, by a theorem of Silvermann [5, Theorem 20.3], which says the specialization map is injective for all but finitely many points $p \in C$, and by Proposition 3.1 , we obtain easily that the rank of curves which are obtained by the specialization from $\mathcal{E}$ by a rational point $p \in C(\mathbb{Q})$ is $\geq 6$ for all but finitely many cases. Hence we get Theorem 1 .

\section{INDEPENDENCE OF RATIONAL POINTS}

To prove Proposition 3.1, since the specialization map is always a homomorphism, we have only to show that there exists a rational point $p$ on $C$ such that $P_{1}, \ldots, P_{6}$ are specialized to six independent rational points on the elliptic curve obtained by the specialization from $\mathcal{E}$ by $p$. We claim this is the case for $p=(272 / 79,11067 / 26)$. Now, we consider the case that $E^{*}$ is the elliptic curve obtained by the specialization $(T, S) \rightarrow(272 / 79,11067 / 26)$ from $\mathcal{E}$. Let the $p_{i}^{*}$ 's be the rational points on $E^{*}$ obtained by the above specialization from $P_{i}$. The equation of $E^{*}$ and the rational points $p_{i}^{*}$ 's are written as follows (for simplicity, we change the coordinate $(1008 / 38950081) \cdot Y$ to $Y)$ :

$$
\begin{aligned}
E^{*}: Y^{2} & =10817567046049 X^{4}-339753752030234 X^{2}+3686523169893001, \\
p_{1}^{*} & =(351 / 79,34570084), \\
p_{2}^{*} & =(430 / 79,-55818951), \\
p_{3}^{*} & =(509 / 79,90688524), \\
p_{4}^{*} & =(123 / 79,-54096988), \\
p_{5}^{*} & =(202 / 79,43904487), \\
p_{6}^{*} & =(439 / 79,-59247156) .
\end{aligned}
$$

Lemma 4.1. Let $E^{*}: Y^{2}=a^{2} X^{4}+b X^{2}+c(a, b, c \in K)$ be an elliptic curve over a field $K$. Then $E^{*}$ is $K$-isomorphic to the curve $E: Y^{2}=X\left(X^{2}-2 b X+b^{2}-4 a^{2} c\right)$, which has a nontrivial rational 2-torsion $(0,0)$, by the map $\phi: E^{*} \rightarrow E$,

$$
\phi(X, Y)=\left(-2 a Y+2 a^{2} X^{2}+b, 4 a^{2} X Y-4 a^{3} X^{3}-2 a b X\right) .
$$

(We note that the two points at infinity of $E^{*}$ map respectively to the unique point at infinity and the point of coordinate $(0,0)$ of $E$.)

Proof. See Mordell [4, p.77]. 
We remark that this lemma gives another proof of the fact that $\mathcal{E}_{A}$ has a nontrivial $\mathbb{Q}(C)$-rational 2-torsion point.

Using Lemma 4.1, we see easily that a Weierstrass model of $E^{*}$, which is denoted by $E$, and the rational points $p_{i}=\phi\left(p_{i}^{*}\right)$ can be written as follows:

$$
\begin{aligned}
E: Y^{2} & =X\left(X^{2}+679507504060468 X-44084234209900772519029117440\right), \\
p_{1} & =(-140066013780432,4093620582907949270112), \\
p_{2} & =(668400902705280,-23931679912802873126400), \\
p_{3} & =(-38170471955952,1617755981603108309088), \\
p_{4} & =(68543386187360,-702002032096036284480), \\
p_{5} & =(-487106389903140,8192998933658320758480), \\
p_{6} & =(718064066419488,-26247951601418953547712) .
\end{aligned}
$$

Now, in order to show the independence of $p_{1}, \ldots, p_{6}$ on $E$, we need notation and two lemmas. Let $E: Y^{2}=X^{3}+a X^{2}+b X$ be an elliptic curve over $\mathbb{Q}$. Then $E$ is 2-isogenous to $E^{\prime}: Y^{2}=X^{3}-2 a X^{2}+\left(a^{2}-4 b\right) X$ by the map $\psi: E^{\prime} \rightarrow E$, $\psi(x, y)=\left(y^{2} / 4 x^{2}, y\left(a^{2}-4 b-x^{2}\right) / 8 x^{2}\right)$. Let $\alpha: E(\mathbb{Q}) \rightarrow \mathbb{Q}^{*} / \mathbb{Q}^{* 2}$ be the map defined by

$$
\alpha(P)= \begin{cases}1 \cdot \mathbb{Q}^{* 2} / \mathbb{Q}^{* 2} & \text { if } P=\infty, \\ b \cdot \mathbb{Q}^{* 2} / \mathbb{Q}^{* 2} & \text { if } P=(0,0), \\ x \cdot \mathbb{Q}^{* 2} / \mathbb{Q}^{* 2} & \text { if } P=(x, y), P \neq \infty,(0,0),\end{cases}
$$

and $\alpha^{\prime}: E^{\prime}(\mathbb{Q}) \rightarrow \mathbb{Q}^{*} / \mathbb{Q}^{* 2}$ the map defined by

$$
\alpha^{\prime}(P)=\left\{\begin{array}{l}
1 \cdot \mathbb{Q}^{* 2} / \mathbb{Q}^{* 2} \text { if } P=\infty \\
\left(a^{2}-4 b\right) \cdot \mathbb{Q}^{* 2} / \mathbb{Q}^{* 2} \text { if } P=(0,0), \\
x \cdot \mathbb{Q}^{* 2} / \mathbb{Q}^{* 2} \text { if } P=(x, y), P \neq \infty,(0,0) .
\end{array}\right.
$$

(We consider $\mathbb{Q}^{*} / \mathbb{Q}^{* 2}$ as a vector space over $\mathbb{Z} / 2 \mathbb{Z}$.)

In the following, we assume that $E(\mathbb{Q})_{\text {tor }}=E^{\prime}(\mathbb{Q})_{\text {tor }}=\{\infty,(0,0)\}$.

Lemma 4.2. The $\mathbb{Q}$-rank of $E$ is equal to

$$
\operatorname{rank}_{\mathbb{Z} / 2 \mathbb{Z}}(\alpha(E(\mathbb{Q})))+\operatorname{rank}_{\mathbb{Z} / 2 \mathbb{Z}}\left(\alpha^{\prime}\left(E^{\prime}(\mathbb{Q})\right)\right)-2 .
$$

Proof. See Zimmer [7, Theorem 8.1].

More precisely, we easily obtain the following lemma.

Lemma 4.3. Let $G$ be a subgroup of $E(\mathbb{Q})$. Then the $\mathbb{Q}$-rank of $G$ is greater than, or equal to, $\operatorname{rank}_{\mathbb{Z} / 2 \mathbb{Z}}(\alpha(G))+\operatorname{rank}_{\mathbb{Z} / 2 \mathbb{Z}}\left(\alpha^{\prime}\left(\psi^{-1}(G)\right)\right)-2$.

We apply Lemma 4.3 to our curve $E$ and the subgroup $G=\left\langle(0,0), p_{1}, p_{2}, \ldots, p_{6}\right\rangle$. In this case, the equation of $E^{\prime}$ is written as

$$
Y^{2}=X\left(X^{2}-1359015008120936 X+638067384914090025583516848784\right) .
$$

We see easily that $E(\mathbb{Q})_{\text {tor }}=E^{\prime}(\mathbb{Q})_{\text {tor }}=\{\infty,(0,0)\}$ by Zimmer [7, Theorem 7.3]. Thus, the assumption of Lemma 4.3 holds. 
By a direct calculation we have

$$
\begin{aligned}
& \alpha((0,0))=-2 \cdot 5 \cdot 7 \cdot 19 \cdot 47 \cdot 67 \cdot 83 \cdot 139 \cdot 181 \cdot \mathbb{Q}^{* 2} / \mathbb{Q}^{* 2}, \\
& \alpha\left(p_{1}\right)=-19 \cdot 79 \cdot 83 \cdot 139 \cdot \mathbb{Q}^{* 2} / \mathbb{Q}^{* 2}, \\
& \alpha\left(p_{2}\right)=2 \cdot 3 \cdot 5 \cdot 47 \cdot 79 \cdot 83 \cdot 181 \cdot \mathbb{Q}^{* 2} / \mathbb{Q}^{* 2}, \\
& \alpha\left(p_{3}\right)=-3 \cdot 7 \cdot 19 \cdot 67 \cdot 79 \cdot 181 \cdot \mathbb{Q}^{* 2} / \mathbb{Q}^{* 2}, \\
& \alpha\left(p_{4}\right)=2 \cdot 5 \cdot 7 \cdot 19 \cdot 47 \cdot 79 \cdot 139 \cdot \mathbb{Q}^{* 2} / \mathbb{Q}^{* 2}, \\
& \alpha\left(p_{5}\right)=-3 \cdot 5 \cdot 7 \cdot 47 \cdot 67 \cdot 79 \cdot 83 \cdot \mathbb{Q}^{* 2} / \mathbb{Q}^{* 2} .
\end{aligned}
$$

So they are independent elements in the $\mathbb{Z} / 2 \mathbb{Z}$-vector space $\mathbb{Q}^{*} / \mathbb{Q}^{* 2}$. On the other hand, let

$$
p^{\prime}=(32608658554556738404 / 169,185553135139334125323174897696 / 2197)
$$

be the rational point on $E^{\prime}$ such that $\psi\left(p^{\prime}\right)=p_{1}+p_{2}+p_{3}+p_{4}+p_{5}+p_{6}$. Then we have

$$
\begin{aligned}
& \alpha^{\prime}\left(p^{\prime}\right)=627169 \cdot \mathbb{Q}^{* 2} / \mathbb{Q}^{* 2}, \\
& \alpha^{\prime}((0,0))=17 \cdot 7103 \cdot 48679 \cdot 627169 \cdot \mathbb{Q}^{* 2} / \mathbb{Q}^{* 2} .
\end{aligned}
$$

So they are independent in $\mathbb{Q}^{*} / \mathbb{Q}^{* 2}$. Using Lemma 4.3 , we can now conclude that $p_{1}, \ldots, p_{6}$ are independent points on $E$, and the proof is complete.

Remark. Using the computer system PARI, we can compute the determinant of the matrix of height pairings $\left\langle p_{i}, p_{j}\right\rangle(1 \leq i, j \leq 6)$. Since this determinant is $48107.7640 \ldots$, the points $p_{1}, \ldots, p_{6}$ are independent on $E$, which gives another proof of Proposition 3.1 .

\section{ACKNOWLEDGMENT}

I should like to express my thanks to Professor Yoshio Mimura at the Osaka Electro and Communication University and Professor Noburo Ishii at the Osaka Prefecture University for their useful advice.

\section{REFERENCES}

1. T. J. Kretschmer, Construction of elliptic curves with large rank, Math. Comp. 46 (1986), 627-635. MR 87g:11069

2. B. Mazur, Rational points on modular curves, Lecture Notes in Math. 601 (1977), 107-148. MR 56:8579

3. J. -F. Mestre, Rang de courbes elliptiques d'invariant donné, C. R. Acad. Sci. 314 (1992), 919-922. MR 93e: 11075

4. L. J. Mordell, Diophantine equations, Academic Press, London, 1969. MR 40:2600

5. J. H. Silverman, The arithmetic theory of elliptic curves, Graduate Texts in Math. 106, Springer-Verlag, New-York, 1986. MR 87g:11070

6. H. G. Zimmer and U. Schneiders, The rank of elliptic curves upon quadratic extension, in Computational Number Theory (eds. A. Pethö, M. E. Pohst, H. C. Williams, H. G. Zimmer), Walter de Gruyter, Berlin, 1991, pp.239-260. MR 92m:11053

7. H. G. Zimmer, Computational aspects of the theory of elliptic curves, in Number theory and applications (ed. R. A. Mollin), Kluwer Academic Publishers, Dordrecht, 1989, pp.279-324. MR 92g:11057

Shiga Polytecnic College, 1414 Furukawa Cho, Oh-Minachiman Shiga 523, Japan

E-mail address: nagao@shiga-pc.ac.jp 